\title{
SU UNA POSSIBILITÀ D'IMPIEGO DEL NEFELOMETRO
}

\author{
Ferruccio Mosetti
}

Da qualche anno è in funzione presso l'Osservatorio Geofisico di Trieste un nefelometro costruito dal Cialdea (1). Esso, come proposto da questo Autore è rivolto a Nord e il suo asse ottico è inclinato di $45^{\circ}$ sull'orizzonte. Solamente se il tratto di cielo esaminato è sereno la registrazione ottenuta col nefelometro è del tipo della fig. 1 . Quando aumenta la nebulosità aumenta di solito l'altezza del nefogramma e diminuisce quella della fascia polarizzata; inoltre la registrazione non è più liscia e regolare ma presenta svariate punte di forma e aspetto diverso a seconda del tipo di nuvolosità : da ciò liı possibilità già mostrata da Cialdea di classificare le nubi mediante la forma del nefogramma.

Non è su questo argomento che ci vogliamo fermare, bensì sull'osservazione, che, detto $p$ il rapporto $\frac{P}{P+N}$, ove $P$ è l'intensità della luce polarizzata (altezza della fascia) e $N$ l'intensità della luce naturale (ordinata dal bordo inferiore del nefogramma), tale grandezza varia con l'umidità dell'aria. Già lo Smosarski $\left({ }^{2}\right)$ aveva notato che la percentuale di luce polarizzata decresceva coll'aumentare della tensione di vapore, ma forse per la scarsità di dati a sua disposizione non aveva mostrato la legge che lega queste due grandezze e non si era soffermato su questo interessante argomento.

Dall'insieme delle nostre registrazioni abbiamo potuto ricavare un centinaio di valori di $p$ con umidità variabile dal $20 \%$ al $90 \%$ e con essi abbiamo disegnato il diagramma di fig. 2. Purtroppo, poiché $\mathrm{i}$ dati in nostro possesso non sono ancora molti e ci mancano sia prove sperimentali che calcoli teorici in merito, argomento questo che ci proponiamo di affrontare in un secondo tempo, non siamo in grado di dare l'equazione esatta della curva da noi empiricamente Irovata, comunque, per lo scopo che qua mostreremo basta anche conoscere l'andamento approssimato di $p$ in funzione di $u$.

Si sa che l'intensità della luce polarizzata diffusa da un punto della volta celeste varia con la posizione del sole rispetto a questo punto, posizione che varia, oltre che durante la giornata anche con 


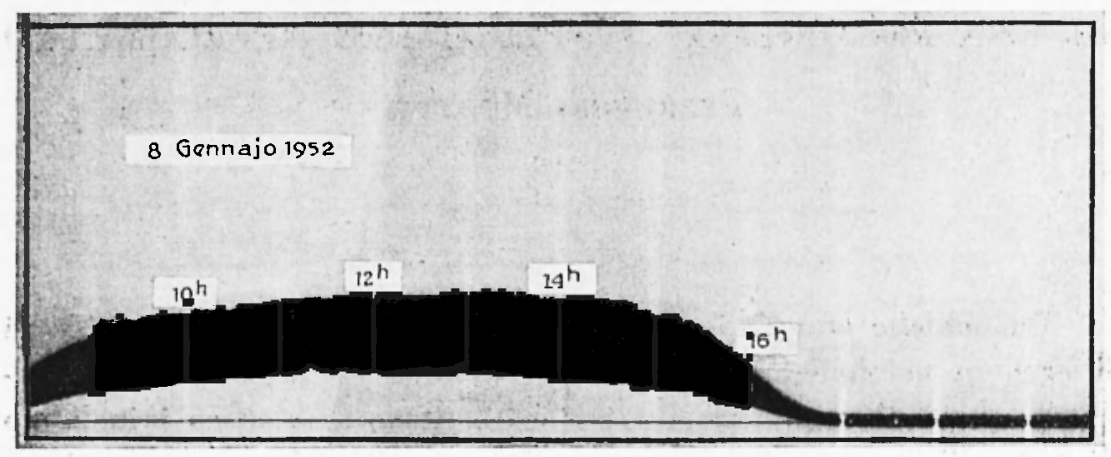

Fig. 1. - Nefogrammat normale oltentilo con cielo sereno.

le stagrioni; il Cialdea nel suo lavoro (") dà dei grafici per tener conto di tali variazioni. Noi però abbiamo visto che non vi è, a parità di umidità variazione apprezzalile di $p$ nel corso della giornata e, anche col variare delle stagioni tale differenza ̀̀ molto piccola, più piccola forse di quella nota teoricamente, e, per il nostro scopo, per cui interessano variazioni molıo più elevate, può anche venir Irascurata. Comunque abbiamo voluto separare $i$ valori di $p$ ottenuti nei mesi primaverili e autunnali e quelli riguardanti $i$ mesi estivi e invernali con i quali abbiamo tracciato i diagrammi parziali di fig. 3 ( 4 rispettivamente.

La curva di fig. 2 rappresenta, come si diceva, l'andamento medio di $P$ in funzione dell'umidita al suolo, ricavalo da valori suscet-

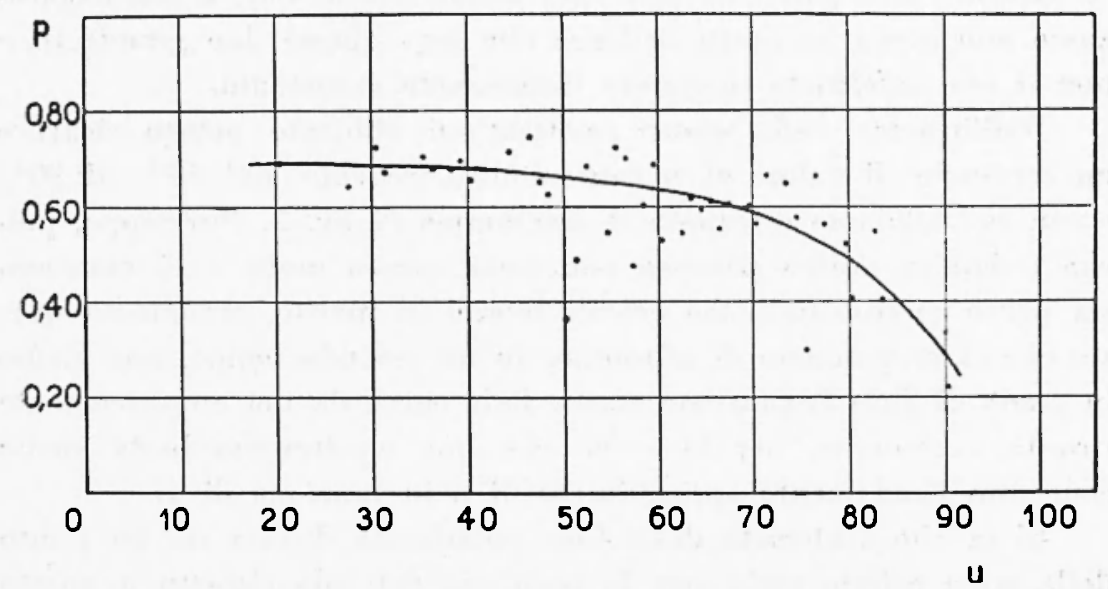

Fig. 2. Diagramma di $\mathrm{p}=\frac{P}{P+N}$ in funzione dell'umiditi percentuale. 
tibili di scarti anche molto forti: è proprio su questi scarti che ci vogliamo soffermare. Succede clue, quando il valore di $p$ che si legge al nefogramma, posto sull'ascissa della fig. 2 corrispondente all'umidità letta al suolo, sta sotto la curva, esso corrisponderebbe ad un'umiditá "fittizia " più o meno maggriore, e inversamente, $\mathrm{i}$ valori di $p$ superiori a quello dell'andamento medio corrisponderebbe ad umidità minori. Pensiamo che molto probabilmente queste anomalie siano dovute ad una ineguale distribuzione del vapore acqueo nella atmosfera. Soltanto se la quantità di vapore è omogeneamence distriluita e decresce uniformemente con l'altezza, seguendo la nota legrge esponenziale, i valori di $p$ ricavati dal nefogramma sono quelli dati dal diagramma di liğ. 2. Se invece in quota vi sono strati di umidità

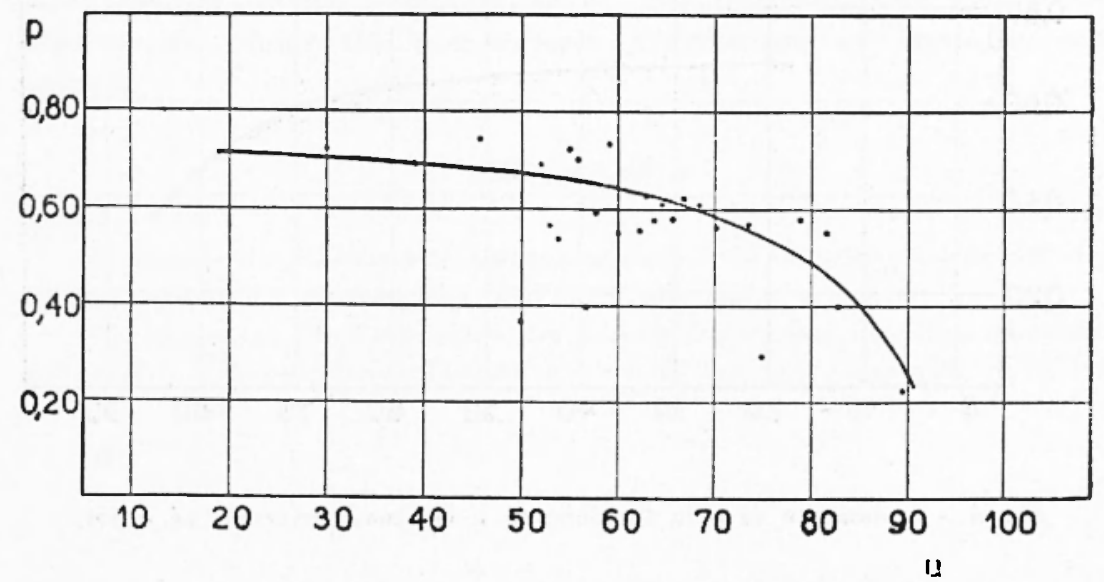

Fig. 3. - Andamento di $p$ in funzione di u nei mesi primaverili e autunnali.

maggiore che al suolo si avrà un assorbimento della luce polarizzata magriore di quello cbe competereblue a questa umidità e si ottiene quindi un valore di $p$ che sta al di sotto della curva. Viceversa un $p$ più alto del normale dovrebbe corrispondere ad un assorbimento più elevato al suolo che non in quota, cioè alla presenza di aria più umida stazionante al suolo.

Formulata questa ipotesi abliamo cercato di controllarla con le osservazioni ed abbiamo trovato appunto che $\mathrm{i}$ valori di $p$ che stavano sotto la curva media corrispondevano a gionnate, o a intervalli di tempo anche più brevi, in cui in quota soffiavano venti sciroccali e si accumulavano masse d'aria umida che dovevano poi condensarsi in nubi: di solito prima della pioggia si ricava dai nefogrammi un 
valore di p più basso della norma. I valori di $p$ più alti si hanno invece quando in quota soffia un vento secco oppure quando al suolo si ha una forte evaporazione, come ad esempio dopo le piogge, le nevicate e i periodi di disgelo.

Sarebbe insomma utile continuare delle ricerche verso questo indirizzo. Ogni stazione dovrebbe anzitutto determinare il valore " normale" di $p$ (che è quello ottenuto con aria secca) e che varia di luogo in luogo, esso è per esempio di 0,70 a Trieste, di 0,80 a

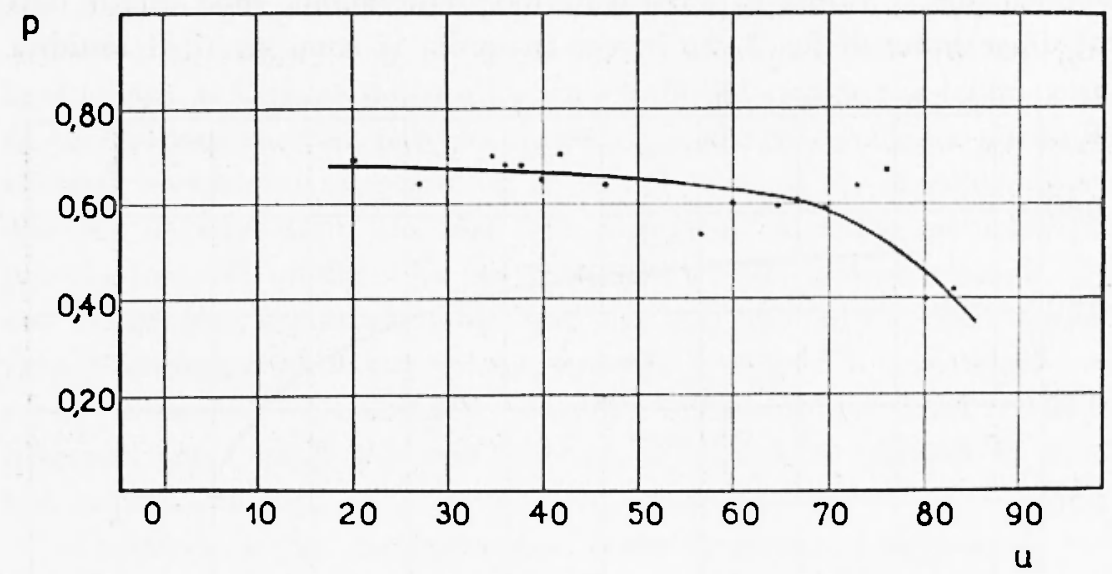

Fig. 4. - Andamento di $p$ in funzione di u nei mesi invernali ed estivi.

Roma (1). Per lo scopo da noi indicato andrebbero usati solamente i nefogrammi normali (come quello della fig. 1) non quelli alterati nell'ampiezza della luce naturale, che corrispondono già ad una maggiore intensità nella diffusione di questa provocata dalla condensazione delle particelle in veli nebulosi più o meno spessi anche se invisibili al nostro occhio; tanto meno sono da usarsi i nefogrammi in cui l'ampiezza della luce polarizzata è molto attenuata o addirittura nulla, i quali corrispondono a strati di particelle ancor più spessi o addirittura a nubi che, oltre a diffondere ancor più la luce naturale, assorbono quasi completamente quella polarizzata.

Istituto Naz. di Geofisica - Osserv. di Trieste - giugno 1952. 


\section{RIASSUNTO}

Si presenta il diagramma di una legge empirica tra la luce polarizzata diffusa dall'atmosfera e l'umidità percentuale di questa; si dà inoltre atto di come, in base a questo diagramma, possano ricavarsi informazioni sull'ineguale distribuzione dell'umidità con l'altezza.

\section{SUMMARY}

A diagram shouing the empirical law between polarized light diffused by the atmosphere and the percent humidity is presented. It is further shown how, by using this diagram, one can obtain information about the non-uniform distribution of humidity with altitude.

\section{BIBLIOGRAFIA}

(1) Cialdea R.: Rilevamento sistematico dello stato di polarizzazione del cielo. Ricerca scientifica e ricostruzione, 1947, n. 5, pag. 633.

(2) Smosarski: Die Polarisation des Himmelslichtes und ihre Zusammenhänge mit anderen Meteorologischen klementen. Gerland Beitr. zur Geophysik, n. 30, 1931. 